\title{
Moderation of severity of audiogenic seizures in DBA/2 mice following intraperitoneal insertion*
}

\author{
EDWARD J. RICHARDSON and EDWARD C. SIMMEL \\ Miami University, Oxford, Ohio 45056
}

Twenty-one-day-old DBA/2J mice, given a blank IP insertion immediately prior to receiving audiogenic seizure stimuli, had a significantly longer latency to Stage 4 seizures (death) than did control mice. A smaller proportion (44\%) IP-insertion animals reached Stage 4 than did controls (80\%).

The interpretation of the physiological and behavioral effects of a drug becomes difficult if the procedure used for the administration of the drug influences these effects in the same direction as that hypothesized for the drug itself. For example, a positive correlation between blood alcohol levels and susceptibility to audiogenic seizures (AGS) was found by Greenberg \& Lester (1953), using stomach tube administration of alcohol, and by Dember, Ellen, \& Kristofferson (1953), using IP injections of alcohol. In both cases, alcohol was assumed to reduce emotionality and thus decrease susceptibility to AGS. However, this paper reports the finding that the mere IP insertion of a 23-ga hypodermic needle significantly moderates the severity of AGS in inbred DBA/2J mice.

\section{METHOD}

The 19 Ss were 21-day-old male DBA/2J mice, first or second generation offspring of breeding pairs obtained from the Jackson Laboratory. DBAs were used because of their well-established high susceptibility to AGS; they were tested when 21 days of age because at this age AGS susceptibility risk (90\%) is highest (Fuller \& Sjursen, 1967; Fuller \& Collins, 1968). Weights of the mice ranged from 6 to $13.5 \mathrm{~g}$.

On the day of testing, the mice were taken from their litters, weighed, and assigned randomly to either the IP insertion group $(\mathrm{N}=9)$ or the control group $(\mathrm{N}=10)$. Both groups contained animals from several different litters. For the experimental animals, an IP insertion was made with a 23-ga hypodermic needle, but nothing was injected during the insertion. Control animals were handled in the same manner, but no IP insertion was made.

Immediately following the insertion or handling, each animal was placed into an IAC acoustic chamber $(61 \times 41 \times 36 \mathrm{~cm})$ for 5 min. Then a bell, located inside the acoustic chamber and producing a measured $100-\mathrm{dB}$ sound, was turned on for a $1-\mathrm{min}$ interval, during which time the onset of the various AGS stages was observed through a one-way window in the front of the acoustic chamber and recorded on an event recorder.

The four stages of AGS were defined as follows: Stage 1-wild running characterized by frenzied running and jumping around the boundary of the chamber; Stage 2-clonic convulsion, during which the animals fell on their sides and drew up their rear legs towards the chin; Stage 3-tonic seizures, during which all four legs were extended caudally; and Stage 4-death. Time of onset

*Reprint requests should be addressed to: Edward C. Simmel, Department of Psychology, Miami University, Oxford, Ohio 45056 . of each stage was defined as follows: Stage 1-when wild running began, Stage 2-when legs began to be drawn up toward chin, Stage 3-when legs began to be extended caudally, and Stage 4-when legs dropped slightly after being extended caudally.

Animals which did not die after exposure to the bell were returned to individual cages for 3 days.

\section{RESULTS AND DISCUSSION}

It was found, as shown in Fig. 1, that the mere insertion of a hypodermic needle not only increased the latency to Stage 4 (death), but that fewer of the animals in the insertion group died ( $44 \%$ vs $80 \%$ of the control animals). A Mann-Whitney U test of the differences of latency of Stage 4 onset between IP insertion and control animals was highly significant $(p<.001)$. A rank-order correlation between the weights of the animals and the latency of Stage 4 onset was run, with any animals not reaching Stage 4 scored for a latency of $60 \mathrm{sec}$. The obtained rho of -0.37 was not significant $(p>.10)$. If weight can be taken as a measure of maturation level, the modification of susceptibility to AGS does not appear to be related to maturation.

None of the mice surviving the first AGS exposure died during the succeeding 3 days, and all of them increased their body weights by at least $15 \%$.

All surviving Ss (those not reaching Stage 4) reached Stage 3 and remained in that stage until the bell ceased ringing. There was no significant difference between surviving and nonsurviving animals, or between experimental and control animals on latency to onset of Stage 3.

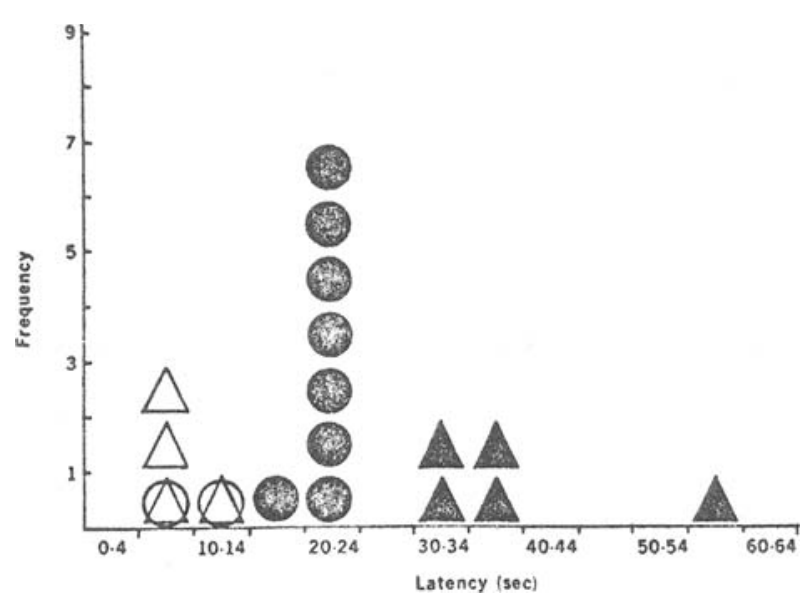

Fig. 1. Latency to onset of highest stage of audiogenic seizure reached. Each triangle represents an IP insertion animal, each circle represents a control animal; each solid symbol represents an animal not surviving (reached Stage 4), each open symbol represents an animal surviving (did not reach Stage 4). 
Insertion animals who survived the AGS procedure did not show a consistent behavioral pattern. One insertion animal, after reaching Stage 3, regressed back through Stage 2 and finally back to Stage 1 type of behavior. Other surviving insertion animals appeared to go from Stage 3 back to Stage 1 without showing any indication of going back into Stage 2 behavior.

After returning to Stage 1, the surviving insertion animals showed a different type of wild running behavior than they did the first time they were in that stage. The second Stage 1 consisted mainly of running and did not show as much jumping as the original Stage 1 behavior. Two animals appeared to have difficulty in standing. When the 1-min bell presentation period ended, the mice would sit "frozen" in a corner of the chamber. Upon removal from the acoustic chamber, the animals were placed in individual home cages. Their behavior in the home cages appeared normal.

Three days following the initial AGS exposure, two of the four surviving insertion-group animals were retested under insertion conditions, and the other two tested under the control condition. One of the two surviving control Ss was tested under the IP condition and the other one retested under the control condition. All six animals reached Stage 4, with no consistent differences in latency of onset. From this, it seems likely that the moderation of severity of AGS by IP insertion is age-dependent.

The results suggest that caution may be advised in interpreting behavioral effects of drugs unless controls for the method of administering are incorporated into the design. In a paper published after this study was completed, Stein (1972) found that blank IP insertions over several days impaired complex maze learning in rats. This could mean that IP insertion effects may be more pervasive than was shown here.

\section{REFERENCES}

Dember, W. N., Ellen, P., \& Kristofferson, A. B. The effects of alcohol on seizure behavior in rats. Quarterly Journal of Studies on Alcohol, 1953, 14, 390-394.

Fuller, J. L., \& Collins, R. L. Temporal patterns of sensitization for audiogenic seizures in SJL/J mice. Developmental Psychobiology, 1968, 1, 185-188.

Fuller, J. L. , \& Sjursen, F. H. Audiogenic seizures in eleven mouse strains. Journal of Heredity, 1967, 58, 135-140.

Greenberg, L. A., \& Lester, D. The effect of alcohol on audiogenic seizures of rats. Quarterly Journal of Studies on Alcohol, 1953, 14, 385-389.

Stein, D. G. The effects of saline or blank injections during development on maze learning at maturity. Developmental Psychobiology, 1972, 5, 319-322.

(Received for publication March 22, 1973.) 